\title{
INMERSIÓN DE SEMILLAS DE MAÍZ EN AGUA CALIENTE EN LA PRODUCCIÓN DE GERMINADOS PARA FORRAJE ${ }^{1}$
}

\author{
Felipe González-Rodríguez $z^{2}$,Lizette Borges-Gómez ${ }^{3}$,Luis Pinzón-López ${ }^{3}$, Miguel Magaña-Magaña ${ }^{3}$, \\ Roberto Sanginés-García ${ }^{3}$, Miguel Urrestarazu-Gavilán ${ }^{4}$
}

\section{RESUMEN}

Inmersión de semillas de maíz en agua caliente en la producción de germinados para forraje. El objetivo de este trabajo fue evaluar la tolerancia al deterioro y la viabilidad de semillas de maíz en inmersión en agua caliente. Se evaluaron tres cultivares de maíz: País Tuxpeño (PT), X'nuuknal (X) y Sinaloa (S) que se sometieron a $0,2,4,6$ y 8 segundos de inmersión en agua a $100^{\circ} \mathrm{C}$, en la producción de germinados para forraje. El estudio se realizó en Conkal, Yucatán, México en junio de 2011. Las variables evaluadas en semillas fueron: peso inicial (PIS), porcentaje de germinación (PG), número medio de días a germinación (NMDG) e índice de velocidad (IVG); en plántulas: peso fresco (PF), seco (PS) y la relación producción de biomasa por unidad de peso de semilla (PF:PIS). Los resultados mostraron diferencias significativas $(\mathrm{P} \leq 0,05)$ en todas las variables, excepto en NMDG. El PG fue de 96,74 y $55 \%$ para PT, X y S, respectivamente. La inmersión por $2 \mathrm{~s}$ incrementó el PG, IVG, PF, PS y PF:PIS en X y S pero con inmersión por 4 y 6 s estas variables disminuyeron. Por el contrario, el PT inmerso por $2 \mathrm{~s}$ disminuyó el PG al 75\%, pero con inmersión por 4 y $6 \mathrm{~s}$ todas las variables mostraron valores ascendentes. El tiempo de inmersión por $8 \mathrm{~s}$ afectó severamente a los tres cultivares, siendo PT el menos afectado ya que el PF solamente disminuyó el 35\%, mientras que para $\mathrm{X}$ y S el decremento fue de $61 \%$ y $42 \%$ respectivamente. En conclusión, PT presentó mayor tolerancia al deterioro por inmersión en agua caliente y por tanto, es el más recomendado para la producción de germinados para forraje verde.

Palabras clave: Zea mays, calidad de semillas, forraje verde.

\begin{abstract}
Immersion of maize seeds in hot water as a treatment to estimulate sprout production for forage. The objective of this work was to evaluate the tolerance to wear and the viability of seeds in three cultivars of maize. The study was conducted in Conkal, Yucatan, Mexico in June 2011. The cultivars used were País Tuxpeño (PT), X'nuuknal (X) and Sinaloa (S). Samples were subjected to $0,2,4,6$ and 8 seconds of immersion in water at $100^{\circ} \mathrm{C}$ to estimulate germination and development of sprouts. The variables evaluated were initial seed weight (ISW), germination percentage (GP), average number of days to germination (ADG), rate of speed of germination (RSG), fresh weight (FW) and dry (DW) of the germinated and the ratio of biomass production per unit of seed weight (FW:ISW). The PG was 96, 74 and 55\% for $\mathrm{PT}, \mathrm{X}$ and $\mathrm{S}$ respectively. The results showed differences (P $\leq 0.05$ ) for all variables except the ADG. The immersion for $2 \mathrm{~s}$ increase the GP, RSG, FW, DW and FW:ISW in X and S but with immersion for 4 and $6 \mathrm{~s}$ these variables decreased. On the contrary, the PT immersed by $2 \mathrm{~s}$ decreased the PG to $75 \%$, but with immersion for 4 and $6 \mathrm{~s}$ all variables showed ascending values. The time of immersion, for $8 \mathrm{~s}$ severely affected to the three cultivar being PT the less affected because the FW only decreased by $35 \%$ while that for $\mathrm{X}$ and $\mathrm{S}$ the decrease was $61 \%$ and $42 \%$ respectively. In conclusion, PT showed higher tolerance to deterioration caused by immersion in hot water and thus the most recommended for the production of germinated for green forage.
\end{abstract}

Keywords: Zea mays, quality of seed, green forage.

\footnotetext{
1 Recibido: 4 de marzo, 2013. Aceptado: 18 de marzo, 2014. Este estudio es parte de la tesis de Doctorado del primer autor, Programa de Doctorado en Ciencias en Agricultura Tropical Sustentable del Instituto Tecnológico de Conkal.

2 Instituto Tecnológico de Conkal, México. delagro2006@yahoo.com.mx

3 División de Estudios de Posgrado e Investigación del Instituto Tecnológico de Conkal. Yucatán, México Km 16.3 antigua carretera MéridaMotul.Conkal, Yucatán México.CP 97345. lizette_borges@hotmail.com (autor para correspondencia), lpinlo@yahoo.com.mx, rsangines@ itaconkal.edu.mx, mmagana@itaconkal.edu.mx

${ }^{4}$ Departamento de Producción Vegetal de la Universidad de Almería, España. mgavilan@ual.es
} 


\section{INTRODUCCIÓN}

El clima de Yucatán, ubicado al sureste de México, se caracteriza por una prolongada época de sequía con una duración de diciembre a junio y una época de lluvias con una duración de junio a noviembre. De acuerdo a Márdero et al. (2012), las proyecciones a cien años indican una disminución de la precipitación en toda Centro América. Específicamente para Yucatán se estima una disminución del $30 \%$ de lluvias, por lo que los tiempos de sequía serán más intensos. Una alternativa para apoyar diferentes sistemas de producción animal es la producción de forraje verde hidropónico (FVH) como alimento de bajo costo. Diferentes especies vegetales han sido utilizadas para la producción de FVH (Vargas, 2008; Romero, 2009; Fuentes et al. 2011); sin embargo, la elección de estas depende de varios factores, entre estos el costo y la disponibilidad en el mercado.

El germinado de maíz utilizado como forraje verde es una alternativa de nutrición animal complementaria, sobre todo en época de sequía; de acuerdo con algunos estudios como el de Sánchez et al. (2010), los resultados obtenidos utilizando FVH de maíz en conejos fueron favorables; también García-Carrillo et al. (2013) evaluaron el FVH de maíz para incrementar la producción de leche de cabra obteniendo resultados satisfactorios.

La calidad de las semillas es determinante para producir los máximos volúmenes de biomasa del forraje y conservar su alto valor nutritivo; característica que se describe por la sumatoria de los atributos genéticos, fisiológicos, físicos y sanitarios, responsables de la capacidad y niveles de productividad (Scheeren et al., 2010). Por otra parte, el envejecimiento o deterioro de las semillas es un fenómeno complejo que difiere entre genotipos, es influenciado por factores ambientales y biológicos, y no ocurre uniformemente, aún dentro de un mismo lote con similar manejo postcosecha (McDonald, 1999).

Se ha demostrado que la disminución del potencial fisiológico de las semillas de maíz, es ocasionada por el envejecimiento natural y provoca la merma progresiva de la capacidad germinativa, la velocidad de crecimiento inicial de la plántula y la tolerancia a condiciones adversas (Marcos-Filho y McDonald, 1998). Estos efectos son asociados a las alteraciones bioquímicas durante las primeras horas de imbibición de los tejidos seminales (Cruz et al., 1995) y se expresan primero en el desempeño germinativo y en el vigor de las semillas y posteriormente a nivel de ADN (Marcos-Filho y McDonald, 1998).

Existen diferentes metodologías para evaluar el envejecimiento acelerado de semillas (AOSA, 1983; García de los Santos, 1991; Bennett, 2002). La inmersión en agua caliente de semillas es un procedimiento utilizado para evaluar su capacidad germinativa; esta técnica puede sustituir al método de cámara de envejecimiento acelerado por ser un método económico, de fácil manejo y no requiere de equipos especializado (Méndez-Natera et al., 2007).

Para el desarrollo e innovación de la tecnología de producción de germinado para forraje en el Estado de Yucatán es necesario evaluar la viabilidad que determinen en forma precisa, sencilla y económica la calidad de la semilla a utilizar. Para tales efectos, el objetivo de este trabajo fue evaluar la tolerancia al deterioro y la viabilidad de semillas de maíz en inmersión en agua caliente.

\section{MATERIALES Y MÉTODOS}

Este trabajo se inició el 9 de junio del 2011 y concluyó el 22 de julio del mismo año, en el Instituto Tecnológico de Conkal ubicado en la antigua carretera Mérida-Motul, en el Estado de Yucatán, México; ubicado en el $\mathrm{Km} 16,5$ a $21^{\circ} 02^{\prime} \mathrm{N}$ y $89^{\circ} 29^{\prime} \mathrm{O}$. El clima del municipio es cálido sub-húmedo con lluvias en verano; la temperatura promedio anual es superior a $\operatorname{los} 26^{\circ} \mathrm{C}$ y la precipitación promedio anual es de 800 a $1000 \mathrm{~mm}$.

En el presente estudio se evaluaron tres cultivares de semillas de maíz almacenadas sin control de temperatura y de humedad y comercializadas comúnmente en la región: País Tuxpeño (PT); X'nuuknal (X) y Sinaloa (S). El País Tuxpeño fue adquirido en el municipio de Becal (20²2’21" LN; 9003’03" LO): el maíz X'nuuknal en el municipio de Maxcanú (20³4'59" LN; 9000`03" LO) y el maíz Sinaloa fue adquirido en el municipio de Baca ( $21^{\circ} 06^{\prime} 00^{\prime \prime} \mathrm{LN}$; 89 $\left.9^{\circ} 25^{\prime} 00^{\prime \prime}\right)$. Los dos primeros son comercializados a granel y el último en sacos de $50 \mathrm{~kg}$. Las semillas fueron adquiridas en junio de 2011 y el porcentaje de humedad de estas fue $11,0,10,38$ y $10,16 \%$, respectivamente.

Los tratamientos evaluados fueron diferentes tiempos de inmersión en agua a $100^{\circ} \mathrm{C}(0,2,4,6$ y 8 segundos) de los tres cultivares de maíz. Se tuvo un total 
de quince tratamientos de un arreglo factorial $3 \times 5$ con cinco repeticiones para un total de 75 unidades experimentales las cuales fueron distribuidas en un diseño de bloques completos al azar. Cada unidad experimental estuvo representada por veinticinco semillas utilizando un total de 1875. Posterior a la inmersión, las semillas fueron sembradas utilizando un sustrato de composta orgánica (Cuadro 1) en bandejas de plástico rígido de $37 \mathrm{~cm}$ de ancho, $60 \mathrm{~cm}$ de largo y $3,5 \mathrm{~cm}$ de altura. Una vez sembradas, las bandejas fueron colocadas en el interior de un invernadero de $5,5 \mathrm{~m}$ de altura, con techo semicircular y provisto de ventilación frontal y lateral. Las bandejas de siembra fueron acondicionadas sobre una mesa a una altura de 1,10 m evitando la salida de las raíces por los orificios de drenaje.

Cuadro 1. Características de la composta utilizada en la producción de germinado de maíz. Instituto Tecnológico de Conkal, Municipio de Yucatán, México. 2011.

\begin{tabular}{lcc}
\hline Parámetro & Unidades & Valor \\
\hline $\mathrm{pH}$ & - & 6,48 \\
$\mathrm{C} . \mathrm{E} .(1: 5)$ & $\mathrm{dS} / \mathrm{m}$ & 2,78 \\
$\mathrm{~N}$ total & $\%$ & 2,24 \\
$\mathrm{P}$ total & $\%$ & 0,31 \\
$\mathrm{~K}$ total & $\%$ & 0,48 \\
$\mathrm{Ca}$ total & $\%$ & 8,7 \\
$\mathrm{Mg}$ total & $\%$ & 0,45 \\
$\mathrm{Na}$ & $\mathrm{mg} / \mathrm{kg}$ & 788 \\
$\mathrm{Cu}$ & $\mathrm{mg} / \mathrm{kg}$ & 17,6 \\
$\mathrm{Fe}$ & $\mathrm{mg} / \mathrm{kg}$ & 5646 \\
$\mathrm{Zn}$ & $\mathrm{mg} / \mathrm{kg}$ & 58,3 \\
$\mathrm{Mn}$ & $\mathrm{mg} / \mathrm{kg}$ & 167,4 \\
\hline
\end{tabular}

El riego se efectuó diariamente mediante un sistema abierto sin recuperación hasta punto de drenaje. A partir de los cinco días de la germinación se aplicó un litro por charola de una solución con $200 \mathrm{mg} / \mathrm{l}$ de N.

La variables para la evaluación de las semillas fueron: peso inicial de la semilla (PIS), para lo cual se registró el peso de cada unidad experimental (25 semillas) utilizando una balanza granataria; porcentaje de germinación (PG), se determinó cuantificando las semillas germinadas a los $3,4,5,6,7,8$ días después de la siembra; el número medio de días a germinación (NMDG) y el índice de velocidad de germinación (IVG), utilizando el modelo propuesto por Maguire (1962):

$$
\begin{gathered}
{\left[N M D G=\frac{(N 1 * T 1+N 2 * T 2+\ldots+N n * T n)}{n}\right]} \\
{[I V G=(N 1 * 4+N 2 * 4+\ldots+N n * 4) / T n]}
\end{gathered}
$$

Donde $N$ es el número de semillas germinadas dentro de los intervalos de tiempo consecutivos; $T$ es el tiempo transcurrido entre el inicio de la prueba $\mathrm{y}$ el fin del intervalo, y $n$ es el número de semillas germinadas.

Las variables evaluadas en las plántulas fueron: peso fresco (PF), que se obtuvo registrando el peso de la plántula completa (vástago + raíz) de todas las semillas germinadas; peso seco (PS), el cual se determinó después de secar las plántulas de doce días después de la siembra en una estufa a $65^{\circ} \mathrm{C} \pm 2$ por tres días; por último se obtuvo la relación producción de biomasa por unidad de peso de semilla (PF:PIS). Los resultados obtenidos fueron analizados estadísticamente con el programa Statgraphic Plus (Fernández-Palacín et al., 2000) realizando análisis de varianza, comparación de medias por Tukey $(\mathrm{p}<0,05)$ y análisis de regresión.

\section{RESULTADOS Y DISCUSIÓN}

El peso inicial de las semillas (PIS) reflejó diferencias significativas $(\mathrm{p} \leq 0,05)$ en los tres cultivares. El mayor PIS se obtuvo en las semillas de Sinaloa $(0,28$ g), seguido de X'nuuknal $(0,25 \mathrm{~g})$ y por último, las semillas País Tuxpeño $(0,22 \mathrm{~g})$.

De acuerdo a la FAO (1993) los tres cultivares de maíz estuvieron en el intervalo de peso inicial, siendo este de 0,190 a 0,300 g por grano. Según Mendoza et al. (2004) y Batistella et al. (2002) las diferencias de peso, tamaño, forma y composición de la semilla pueden deberse a factores genéticos, ambientales y a la ubicación de esta en la mazorca.

\section{Porcentaje de germinación e índice de velocidad de germinación}

El porcentaje de germinación (PG) y el índice de velocidad de germinación (IVG) mostraron diferencias 
significativas entre cultivares, tiempos de inmersión e interacción de ambos $(\mathrm{p} \leq 0,05)$ (Cuadro 2); únicamente en el número medio de días a germinación (NMDG) no hubo respuesta significativa. En promedio todas las semillas germinaron entre los cuatro y cinco días después de la siembra.

En la variable de PG se observó que cuando las semillas no fueron tratadas con agua caliente, el cultivar País Tuxpeño alcanzó el mayor PG con 96\%; para este mismo tiempo las cultivares de $\mathrm{X}^{\prime}$ nuuknal y Sinaloa obtuvieron el 80 y $71 \%$, respectivamente (Figura 1).

En los cultivares X`nuuknal y Sinaloa, cuando las semillas fueron inmersas en agua caliente por $2 \mathrm{~s}$, se tuvo un incremento del 7 y $16 \%$ de PG respectivamente; lo que sugiere que tiempos muy cortos de inmersión en agua caliente puede mejorar la germinación de semillas de menor calidad germinativa. Es probable que estos resultados se deban a un restablecimiento de las membranas celulares en el proceso de germinación, ya que de acuerdo a Gutiérrez et al. (2007) el proceso inicial de hidratación de las semillas ocasiona alteraciones en la funcionalidad de las membranas celulares, misma que se restablece conforme avanza la germinación según sea el vigor de la semilla. El efecto positivo observado en los tiempos de inmersión por $2 \mathrm{~s}$, pueden ser atribuidos a un efecto de hormesis, el cual explica los efectos favorables obtenidos por agentes que en bajas dosis tienen efectos biopositivos pero que en altas dosis tienen un efecto bionegativo (Luckey, 1993). Este fenómeno ha sido observado en semillas de maíz tratadas con extractos inhibitorios del crecimiento (Laynez-Garsaball y Méndez-Natera, 2007).
Por su parte, Méndez et al. (2008) señalan el efecto favorable de la temperatura sobre la permeabilidad del pericarpio. No obstante, incrementos en el tiempo de inmersión en agua caliente redujeron considerablemente el PG, observándose en este estudio que cuando las semillas se sumergen por 6 y $8 \mathrm{~s}$ la germinación decrece, indicando un mayor deterioro en los granos maíz. Este mismo comportamiento ha sido reportado por Méndez et al. (2007), al señalar que a medida que se incrementan los tiempos de inmersión en agua caliente a $100^{\circ} \mathrm{C}$ se provoca un deterioro en la semilla de maíz, originando una baja germinación. De acuerdo a los resultados obtenidos, la semilla de País Tuxpeño presentó el mayor grado de tolerancia al deterioro, ya que, aun con tiempos de inmersión de 8 s, mostró mayor porcentaje de germinación que en las semillas de X'nuuknal y Sinaloa, quienes fueron más susceptibles al tratamiento calórico de 6 y $8 \mathrm{~s}$ de inmersión.

En el índice de velocidad de germinación (IVG), se observó que las semillas germinadas por día disminuyeron con el aumento del tiempo de inmersión para $X$ nuuknal y Sinaloa (Figura 1b). Cuando las semillas no fueron inmersas en agua caliente $(0 \mathrm{~s})$, se observó en País Tuxpeño mayor IVG seguido del X'nuuknal y Sinaloa. El cultivar País Tuxpeño disminuyó su IVG a los $2 \mathrm{~s}$ y posteriormente, se incrementó a medida que aumentó el tiempo de inmersión hasta los $4 \mathrm{~s}$, para luego disminuir conforme aumentó el tiempo de inmersión. Por el contrario, en las semillas X'nuuknal y Sinaloa la inmersión por $2 \mathrm{~s}$ favoreció la velocidad de germinación; sin embargo, tiempos mayores de inmersión provocaron una disminución en el IVG. Tales efectos pueden estar asociados a alteraciones

Cuadro 2. Cuadrados medios y significancia estadística de las variables de germinación de semillas de tres cultivares de maíz inmerso en agua a $100^{\circ} \mathrm{C}$ por tiempos de 0, 2, 4, 6 y 8 s. Instituto Tecnológico de Conkal, Municipio de Yucatán, México. 2011.

\begin{tabular}{lcccc}
\hline Fuente de variación & Variedad & Tiempo & Variedad*Tiempo & Error \\
\hline Grados de libertad & 2 & 4 & 8 & 60 \\
Porcentaje de germinación (PG) & $8012,30^{1}$ & $2188,90^{1}$ & $608,50^{1}$ & 195,20 \\
Valor de F para PG & 41,056 & 11,21 & 3,11 & 0,27 \\
No. medio de días a germinación & 0,22 & 0,56 & 0,45 & 0,63 \\
Índice de velocidad de germinación (IVG) & $25,16^{*}$ & $8,78^{*}$ & $1,83^{*}$ & 2,9 \\
Valor de F para IVG & 40,22 & 14,03 & \\
\hline
\end{tabular}

${ }^{1}$ significativo $(\mathrm{p}<0,05)$. 
a

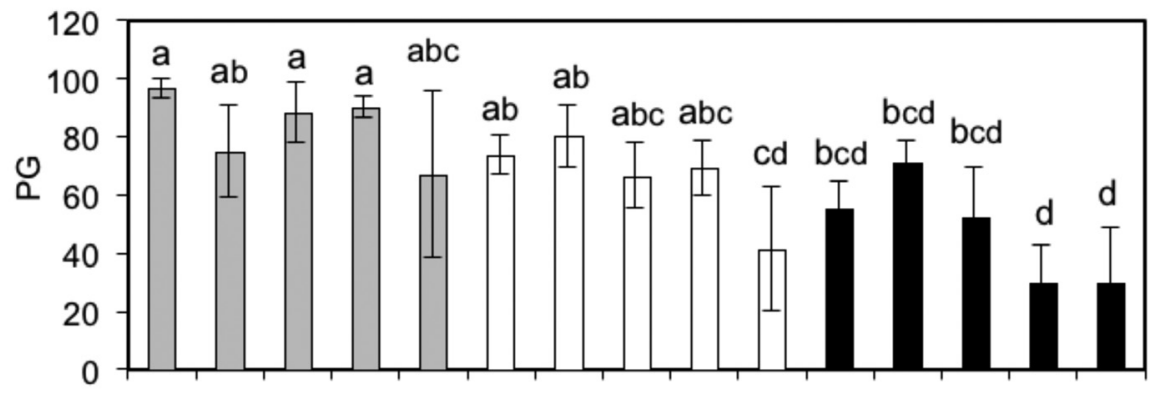

$\mathrm{b}$

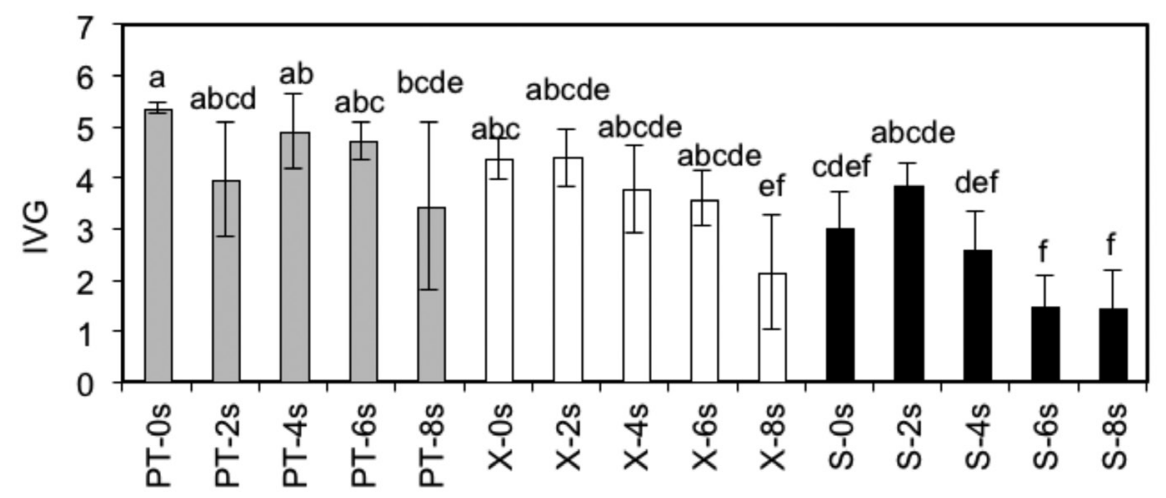

Cultivar de maiz y tiempo de inmersión en agua a $100^{\circ} \mathrm{C}$

Figura 1. (a) Porcentaje de germinación (PG) y (b) índice de velocidad de germinación (IVG) de tres cultivares de maíz ( $\mathrm{PT}=$ País Tuxpeño, $\mathrm{X}=$ Xnuuknal y $\mathrm{S}=$ Sinaloa) sometidos a $0,2,4,6$ y $8 \mathrm{~s}$ de inmersión en agua a $100^{\circ} \mathrm{C}$. Instituto Tecnológico de Conkal, Municipio de Yucatán, México. 2011. Medias con letras iguales son estadísticamente idénticos (Tukey 0,05 ).

en la funcionalidad de membranas, lisis membranal o daños estructurales en el ADN que ocurren durante las primeras horas de imbibición de los tejidos seminales (Cruz et al., 1995). La sensibilidad de las semillas por efecto de los tiempos de inmersión y las variables de germinación PG e IVG mostró una función polinómica (Figura 2) la cual describe la necesidad de aplicar o no el tratamiento de agua a $100^{\circ} \mathrm{C}$ para mejorar la germinación.

Peso fresco y seco de las plántulas y relación producción de biomasa por unidad de peso de semilla

En la evaluación de las variables de crecimiento, el peso fresco (PF), el peso seco (PS), y la relación producción de biomasa por unidad de peso de semilla (PF:PIS), mostraron diferencias significativas $(\mathrm{p} \leq 0,05)$ para los tres cultivares y los tiempos de inmersión, pero no para la interacción de ambos, excepto para la relación PF:PIS donde sí se reflejó una respuesta significativa $(\mathrm{p} \leq 0,05)$ en la interacción (Cuadro 3$)$. Lo anterior indica que existen diferencias marcadas entre genotipos para las características evaluadas, atribuidas a un desarrollo distinto y a un patrimonio genético que se expresó de manera diferencial; aun cuando se proporcionó ambientes similares. Según Sánchez et al. (2011), el genotipo es determinante en las características de crecimiento y producción de forraje de maíz, las cuales pueden ser afectadas por diferentes factores por lo que los tiempos de inmersión tuvieron efectos independientes para cada variedad.

La semilla de mayor peso inicial (Sinaloa) no mostró la mayor producción de PF y PS de plántulas de maíz; por el contrario, siendo la semilla de mayor 


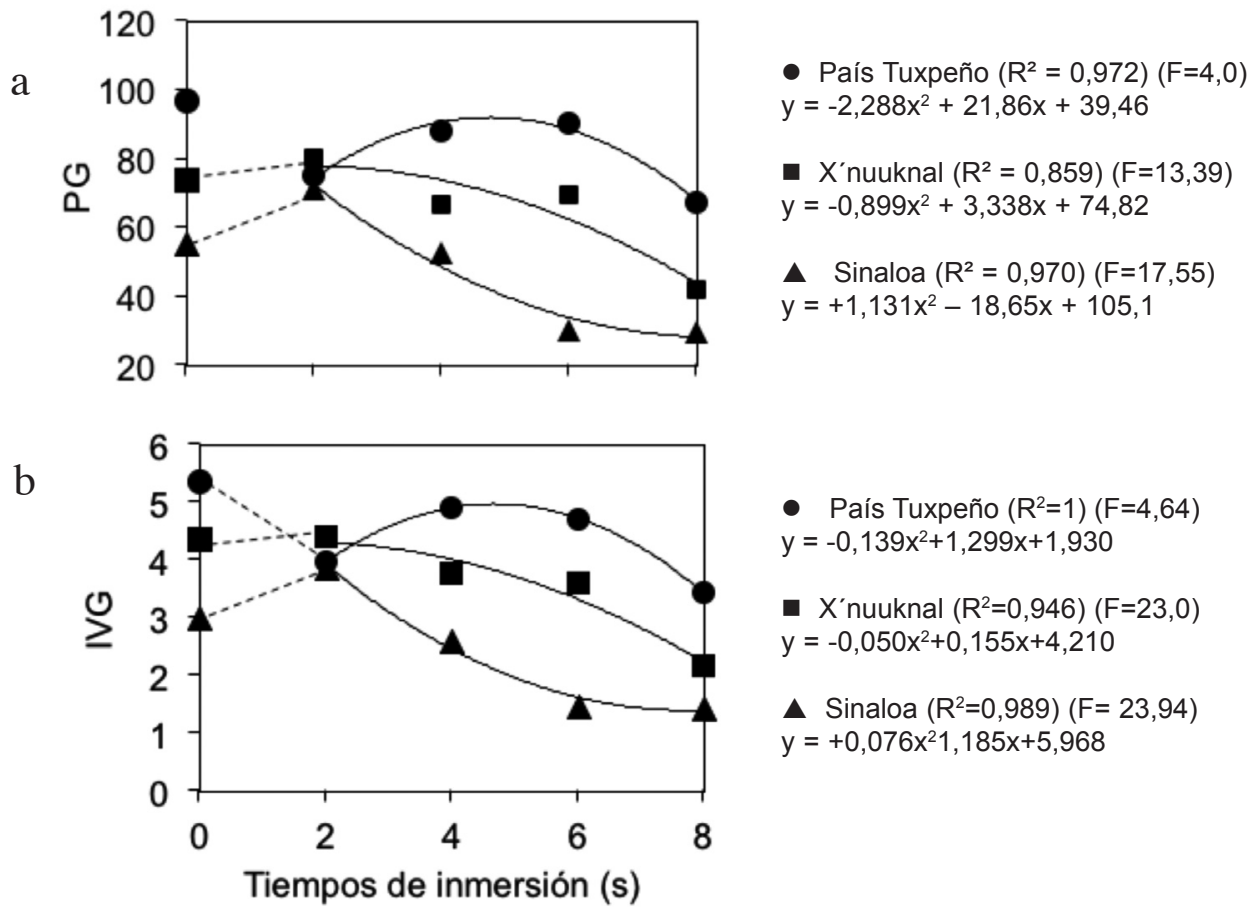

Figura 2. Análisis de Regresión de las variables de a) porcentaje de germinación (PG) y b) índice de velocidad de germinación (IVG) de tres cultivares de maíz y tiempos de inmersión en agua a $100^{\circ} \mathrm{C}$, registrado durante diez días después de la siembra. Instituto Tecnológico de Conkal, Municipio de Yucatán, México. 2011.

Cuadro 3. Cuadrados medios de las variables de crecimiento de tres cultivares de maíz inmerso en diferentes tiempos en agua a $100^{\circ} \mathrm{C}$. Instituto Tecnológico de Conkal, Municipio de Yucatán, México. 2011.

\begin{tabular}{|c|c|c|c|c|}
\hline Fuente de variación & Variedad & Tiempo & Variedad*Tiempo & Error \\
\hline Grados de libertad & 2 & 4 & 8 & 60 \\
\hline Peso fresco de plántula (PF) & $14,83^{1}$ & $1,23^{1}$ & 0,1790 & 0,0876 \\
\hline Valores de F para PF & 169,37 & 14,09 & 119,89 & \\
\hline Peso seco de plántula (PS) & $0,03^{1}$ & $0,004^{1}$ & 0,0008 & 0,0005 \\
\hline Valores de F para PS & 71,466 & 8,744 & 1490,084 & \\
\hline $\begin{array}{l}\text { relación producción de biomasa por } \\
\text { unidad de peso de semilla (PF:PIS) }\end{array}$ & $324,50^{1}$ & $22,27^{1}$ & $3,2830^{1}$ & 1,379 \\
\hline Valores de F para PF:PS & 235,46 & 16,16 & 1474,88 & \\
\hline
\end{tabular}

${ }^{1}$ significativo $(\mathrm{p} \leq 0,05)$.

peso, una menor producción de biomasa fue observada. Algunos autores señalan que en maíz, el rendimiento está más asociado al número de granos que al peso de los mismos ya que dentro de los componentes que dan lugar al rendimiento, el número por unidad de superficie es mucho más variable que el peso del grano (Otegui y Andrade, 2000). En este estudio, el número de semillas fue de 25 semillas por unidad 
experimental; sin embargo, el peso para cada unidad experimental fue diferente. De acuerdo a LópezSantillán (2004), el tamaño es más importante que el número ya que una semilla de menor tamaño concluiría con mayor rapidez la etapa de germinación permitiendo un estado fenológico más avanzado, es probable que a ello se deban los valores encontrados en la semilla de País Tuxpeño.

Cuando las semillas no fueron tratadas con agua caliente, las semillas de País Tuxpeño mostraron los valores más altos de PF y PS (2,24 y 0,158 g/plántula, respectivamente) (Figura 3). La producción de PF y PS de esta variedad, solamente fue afectada cuando las semillas fueron inmersas por un tiempo de $8 \mathrm{~s}$, disminuyendo la producción de las plántulas a un 35 y $26 \%$, respectivamente.

Contrario a lo observado con las semillas de País Tuxpeño, las semillas de X nuuknal y Sinaloa produjeron menor biomasa aun sin haber sido sumergidas en agua caliente; no obstante, al ser inmersas por 2 s se observó un incremento en la producción de biomasa, la cual decreció posteriormente conforme se incrementó el tiempo de inmersión. Se observó que al tiempo de inmersión de $8 \mathrm{~s}$ la disminución de PF y PS de las plántulas fue del 65 y $47 \%$ para las semillas de X nuuknal y del 57 y 53\% para las de Sinaloa, respectivamente. Estos resultados muestran que las semillas de la variedad País Tuxpeño no son susceptibles a una temperatura del agua de $100^{\circ} \mathrm{C}$; por el contrario, las de X nuuknal y Sinaloa son más susceptibles, pero con tiempos breves de inmersión (2 s) puede mejorarse la producción de biomasa. Esto coincide con Méndez (2007), quien menciona que a medida que se someten semillas de maíz a mayores tiempos de inmersión se provoca un deterioro en la semilla que origina bajo porcentaje de germinación y crecimiento de plántulas con menor peso fresco y seco. La sensibilidad de las semillas por efecto de los tiempos de inmersión y la producción de biomasa puede observarse en la relación polinómica de la Figura 4, en donde se muestra

a

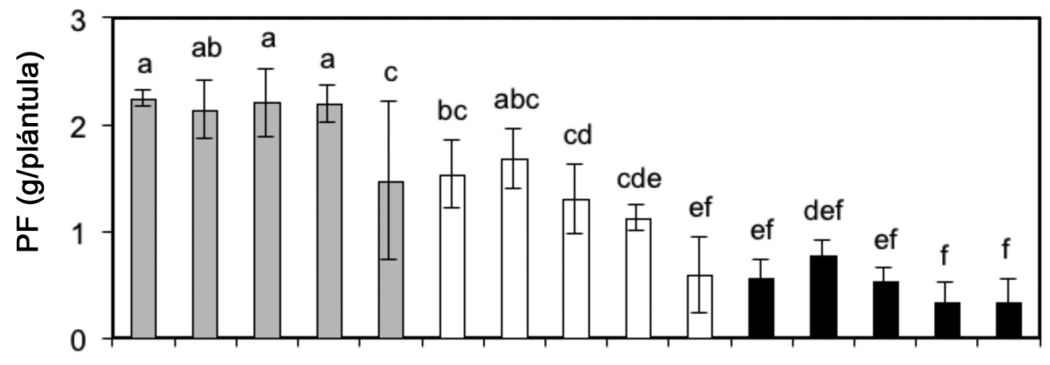

b

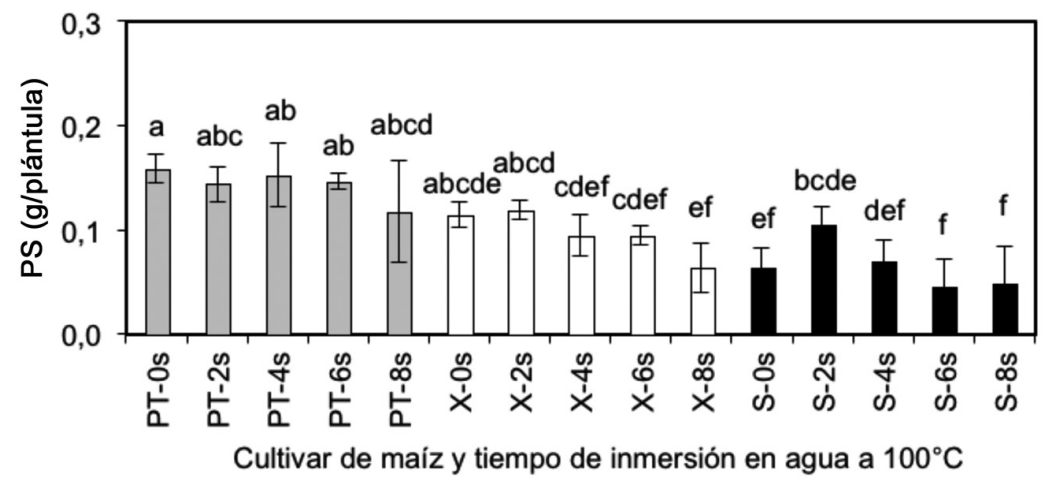

Figura 3. (a) peso fresco (PF) y (b) peso seco (PS) de tres cultivares de maíz (PT = País Tuxpeño, $\mathrm{X}=$ Xnuuknal y $\mathrm{S}=$ Sinaloa) sometidos a 0,2 , 4, 6 y 8 s de inmersión en agua a $\left(100^{\circ} \mathrm{C}\right)$. Instituto Tecnológico de Conkal, Municipio de Yucatán, México. 2011. Medias con letras iguales son estadísticamente iguales (Tukey 0,05). 

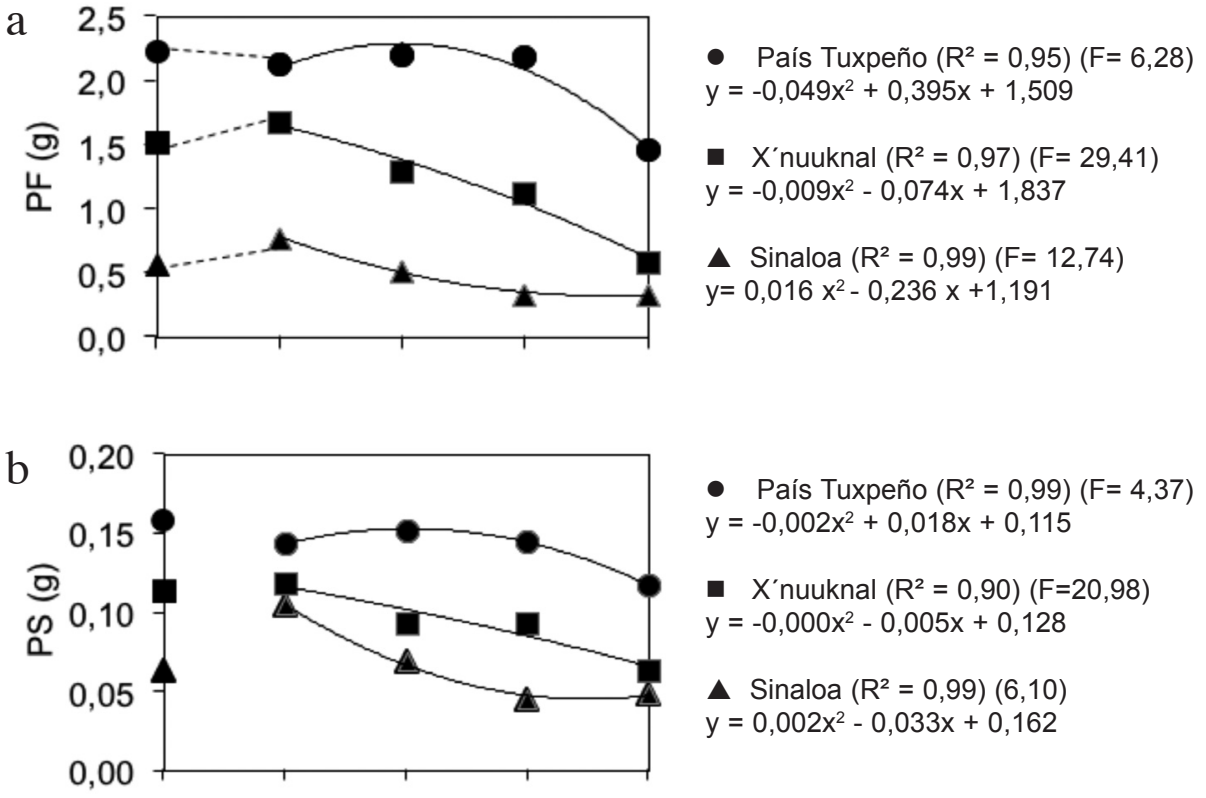

Figura 4. Regresión entre tiempos de inmersión a $0,2,4,6$ y $8 \mathrm{~s}$ en agua a $100^{\circ} \mathrm{C}$ y a) peso fresco (PF) y b) peso seco (PS) a 12 días después de la siembra. Instituto Tecnológico de Conkal, Municipio de Yucatán, México. 2011.

la conveniencia de aplicar el tratamiento de inmersión de las semillas en agua a $100^{\circ} \mathrm{C}$ y los tiempos más convenientes de inmersión.

Al analizar la influencia de los tiempos de inmersión en la relación del PF: PIS, se observó una disminución en estas relaciones para los $8 \mathrm{~s}$ (Figura 5). La mayor producción de biomasa por gramo de semilla la obtuvo la variedad País Tuxpeño con una relación 1:10,05. En X’nuuknal la mayor producción de biomasa por gramo de semilla fue de 1:6,70 cuando

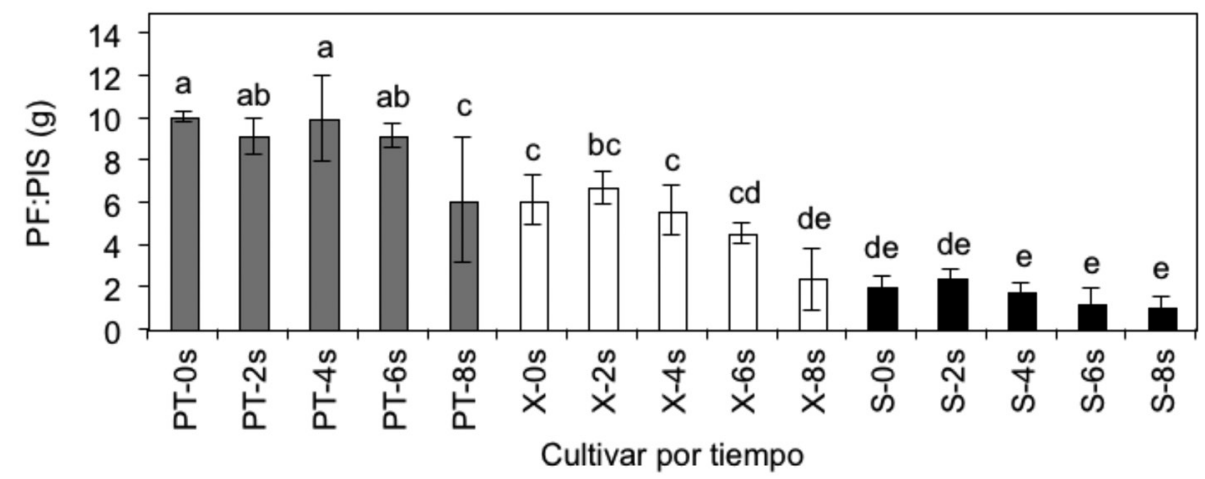

Figura 5. Producción de biomasa fresca por gramo de semilla de tres cultivares de maíz (PT = País Tuxpeño, $\mathrm{X}=$ Xnuuknal y $\mathrm{S}=$ Sinaloa) sometidos a 0, 2, 4, 6 y 8 s. de inmersión en agua a $100^{\circ} \mathrm{C}$. Instituto Tecnológico de Conkal, Municipio de Yucatán, México. 2011. Mismas literales son estadísticamente iguales, Tukey $(\mathrm{p}<0,05)$. 
las semillas fueron sumergidas por $2 \mathrm{~s}$ y para Sinaloa la mejor relación fue 1:2,41 para este mismo tiempo de inmersión.

En la comparación productiva de germinado de forraje verde hidropónico de maíz, arroz y sorgo negro forrajero Vargas (2008), obtuvo una relación de semilla: biomasa fresca para sorgo de 1: 5,45; para maíz una relación de 1:4,3 y para arroz 1:3,58. Elizondo (2005) menciona, que a partir de $1 \mathrm{~kg}$ de los mismos cereales puede llegarse a obtener hasta $9 \mathrm{~kg}$ de biomasa. En este estudio, se atribuye al tiempo de inmersión de 6 y $8 \mathrm{~s}$ el bajo porcentaje de germinación y los menores pesos frescos obtenidos en los tres cultivares de maíz.

\section{LITERATURA CITADA}

AOSA (Association of Official Seeds Analysis). 1983. Seed vigor testing handbook: Contribution No. 32 to the handbook on seed testing. Association of Official Seed Analysts, Washington, USA.

Batistella, F.F., V.F. Moro, y M.N. De Carvalho. 2002. Relationships between physical, morphological, and physiological characteristics of seeds developed at different positions of the ear of two maize (Zea mays L.) hybrids. Seed Sci. Tech. 1(30):97-106.

Bennett, M. 2002. Saturated salt accelerated aging and other vigor tests for vegetable seeds. En: McDonald, y S. Contreras, editores, Proceedings International Seeds Seminar: Trade, Producction and Technology. Pontificia Universidad Católica de Chile, Chile. p. 188-193.

Cruz, G.F., H.V. González, M.J. Molina, y R.J. Vázquez. 1995. Seed deterioration and respiration as related to DNA metabolism in germinating maize. Seed Science and Technology 2(23):477-486.

Elizondo, J. 2005. Forraje verde hidropónico. Una alternativa para la alimentación animal. Revista ECAG Informa (32):36-39

FAO (Organización de las Naciones Unidas para la Agricultura y la Alimentación). 1993. El maíz en la nutrición humana. Colección FAO: Alimentación y Nutrición. No. 25. Roma, Italia.

Fernández-Palacín, F., S.M. López, M.M Muñoz, M. Rodríguez-Chía, A. Sánchez-Navas, y C. ValeroFranco. 2000. Estadística asistida por ordenador: Statgraphics Plus 4.1. Universidad de Cádiz, España.

Fuentes, C.F.F., P.C.E. Poblete, y P.M.A. Huerta. 2011. Respuesta reproductiva de conejos alimentados con forraje verde hidropónico de avena, como reemplazo parcial de concentrado comercial. Acta Agronómica 60(2):183-189.

García de los Santos, G., y L.P. Camargo. 1991. Comparación de tres pruebas para evaluar el vigor de semilla de siete variedades de frijol. Agrociencia 4:131-143.

García-Carrillo, M., L. Salas-Pérez, J.R. Esparza-Rivera, P. Preciado-Rangel, y J. Romero-Paredes. 2013. Producción y calidad fisicoquímica de leche de cabras suplementadas con forraje verde hidropónico de maíz. Agron.Mesoam. 24(1):169-176.

Gutiérrez, H.G., V.J. Virgen, y V.J. Arellano. 2007. Germinación y crecimiento inicial de semillas de maíz con envejecimiento natural. Agron. Mesoam. $18(2): 163-170$.

Laynez-Garsaball, J.A., y J.R. Méndez-Natera. 2007. Efectos de extractos acuosos de la maleza Cyperus rotundus $\mathrm{L}$. (Cyperaceae) sobre la germinación de semillas y crec imiento de plántulas de maíz (Zea mays L.) cv. Pioneer 3031. Rev. Peru. Bio. 14(1):55-60.

López-Santillán, J.A., S. Castro-Nava, C. Trejo-López, M. del C. Mendoza-Castillo, y J. Ortiz-Cereceres. 2004. Biomasa acumulada e intercambio gaseoso en maíz proveniente de semilla de diferente tamaño bajo humedad favorable y restringida. Phyton-International Journal Of Experimental Botany 73:243-248.

Luckey, T.D. 1993. Radiation hormesis: biopositive effect of radiations. GIRI meeting. Momplelier, France. http://link.springer.com/ chapter/10.1007/978-94-011-5804-6_2 (Consultado nov. 1993).

Maguire, J.D. 1962. Speed of germination in selection and evaluation for seedling emergence and vigor. Crop Sci. 2:176-177.

Marcos-Filho J., y M.B. McDonald. 1998. Sensitivity of RAPD analysis, germination and vigor test to detect the intensity of deterioration of naturally and artificially aged soybean seeds. Seed Sci. Technol. 26:141-157.

Márdero, S., E. Nickl, B. Schmook, L. Schneider, J. Rogan, Z. Christman, y D. Lawrence. 2012. Sequías en el sur de la península de Yucatán: análisis de la variabilidad anual y estacional de la precipitación. Investigaciones Geográficas. Boletín del Instituto de Geografía, UNAM 78:19-33.

McDonald, M.B. 1999. Seed deterioration: physiology, repair, and assessment. Seed Sci. Technol. 27:177-237.

Méndez-Natera, J.F., L. Ysavit-Marcano, y J.F. MerazoPinto. 2007. Efecto de la inmersión de semillas de maíz (Zea mays L.) en agua a $100^{\circ} \mathrm{C}$ sobre 
la germinación y crecimiento de plántulas bajo condiciones de laboratorio. TIP Rev. Esp. Cienc. Quim. Biol. 10(2):56-64.

Méndez, N.N., M.L. Ysavit, y P.J. Merazo. 2007. Uso del agua caliente para evaluar la calidad de semillas de maíz (Zea mays L.). Revista Tecnológica ESPOL. 1(20):229-236.

Méndez, N.N., P.J. Merazo, y M.N. Montaño. 2008. Relación entre la tasa de imbibición y el porcentaje de germinación en semillas de maíz (Zea mays L.), caraota (Phaseoulus vulgaris L.) y quinchoncho (Cajanum cajan L.). Revista UDO Agrícola 8(1):61-62.

Mendoza, E.M., M.L. Latournerie, E. Moreno, G. Castañón, J. Cruz, C. De León, J.G. García. 2004. Cambios en la calidad de la semilla de maíz durante su desarrollo y maduración. México. Agron. Mesoam. 15(2):155-160.

Otegui, M.E., y F.H. Andrade. 2000. New relationships between light interception, ear growth and kernel set in maize. En: M.E. Westgate, y K. Boote, editors, Physiology and modeling of kernel set in maize, Crop Sci. Soc. of America y Amer. Soc. of Agronomy Special Publication $\mathrm{N}^{\circ}$ 29. Baltimore, Maryland, EEUU. p. 89-102.
Romero, V.M.E., D.G. Cordoba, y G.E.O. Hernández. 2009. Producción de forraje verde hidropónico y su aceptación en ganado lechero. Acta Universitaria. Universidad de Guanajuato 19(2):11-19.

Sánchez, L.A., Ch.A. Meza, T.A. Álvarez, y Z.L. Rizzo. 2010. Forraje verde hidropónico de maíz (Zea mays) deshidratado en el engorde de conejos Nueva Zelanda (Oryctolagus cuniculus). Ciencia y Tecnología 32(2):21-23.

Sánchez-Hernández, M.A., C.U. Aguilar-Martínez, N. Valenzuela-Jiménez, C. Sánchez-Hernández, M.C. Jiménez-Rojas, y C. Villanueva-Verduzco. 2011. Densidad de siembra y crecimiento de maíces Forrajeros. Agronomía Mesoamericana 22(2):281-295.

Scheeren, B.R., Peske, S.T., Braga, S.L., y Albuquerque, B.A. 2010 Qualidade fisiológica e produtividade de sementes de soja. Revista Brasileira de Sementes 32(3):35-41.

Statgraphic Plus. 1999. Versión 4.1. Dtadistical graphics Corp.

Vargas, R.C. 2008. Comparación productiva de forraje verde hidropónico de maíz, arroz y sorgo negro forrajero. Agron. Mesoam. 19(2):233-240. 